\title{
Heyting Valued Set Theory and Fibre Bundles
}

By

\author{
Hirokazu NishimurA*
}

\begin{abstract}
Alustrien
Takeuti and Titani [15] have demonstrated that, given a manifold $B$ with topology $\Omega=$ $O(B)$, the internal notion of an apartness vector space in $V(\Omega)$ and the external notion of a vector bundle over $B$ are no more than two representations of the same entity. The principal objective of this paper is, first of all, to internalize fibre bundles on the lines of Takeuti and Titani [15], and then to establish various internal-external interconnections around this. For example, we show that the external notion of integration over the fibre corresponds to the usual integration on internalized manifolds within $V(\Omega)$. The paper aitains its climax as we discuss the internal and external aspects of an internalized version of celebrated Stokes theorem.
\end{abstract}

\section{§1. Introduction}

The notion of a fibre bundle plays a significant role in geometry of manifolds and the main purpose of this paper is to demonstrate that many constructions and concepts on fibre bundles have their natural interpretation in Heyting valued set theory. Let $\xi=(E, \pi, \mathbb{R}, \mathbb{F})$ be a smooth fibre bundle, where $\mathbb{E}$ is the total space, $B$ is the base space, $\pi: E \rightarrow B$ is the projection, and $F$ is the typical fibre. In the first place, after reviewing some rudimenis of Heyting valued set theory in Section 2, we show, on the lines of Takeuti and Titani [15], that the total space $E$ can be regarded as a smooth manifold $E^{\infty}$ in $V^{(\Omega)}$ with $\Omega$ being the topology of the base space $B$, which will be done in Section 3. In Section 4 we show that every vector bundle $Z$ over $\mathbb{E}$ can be internalized as a vector bundle $Z^{\infty}$ over $E^{\infty}$ in $V^{(\Omega)}$. As an application of this idea, we will find out what the tangent and orientation bundles of $E^{\infty}$ look like externally. Section 5 is devoted to differential forms with a result on the existence and uniqueness of exterior differentiation in $V^{(\Omega)}$. The first main result of Section 6 is that the well-known external notion of fibre-compact support corresponds to the internal

Communicated by S. Takasu, May 28, 1987. Revised September 16, 1987.

* Institute of Mathematics, University of Tsukuba, Ibaraki 305, Japan. 
notion of compact support, whose definition requires some elaboration, since closed sets are not well-behaved creatures in intuitionistic topology. Then we can define integration of differential forms with compact supports on oriented smooth manifolds in $V^{(\Omega)}$. As an application of this idea, we will show that the well-known external notion of integration over the fibre has its internal counterpart. Just as differential calculus and integral calculus of high school mathematics are related in the fundamental theorem of calculus, Stokes' theorem is the crystalization of relationship between differentiation and integration on manifolds. Thus Section 7 is devoted to Stokes' theorem in $V^{(\Omega)}$. There we will demonstrate that the well-known commutativity of exterior differentiation and integration over the fibre, say,

$$
d \circ f_{F}=f_{F} \circ d
$$

is an external restatement of an internal version of Stokes' theorem in disguise. The final section is reserved for an open problem.

As is well-known, not every classically equivalent definition yields an intuitionistically equivalent definition. Therefore we agree that we should try to make it clear which classical definition we adopt as our internal one. However we will usually neglect to write out the appropriate apartness relation on each occasion, though we prefer apartness. As far as this paper is concerned, to provide such a definition would always be a routine, and its details are often cumbersome both for the author to write out and for the reader to make out. Thus we have chosen to be loose in this matter. Last but not least, since we deal with two set theories $\mathbb{Z F C}$ and $Z_{I}$, we feel it a must to make it explicit which set theory our definitions and theorems are made with respect to, though most of them are made within $\mathbb{Z F C}$.

\section{§2. Intuitionistic Set Theory}

In this section we review some rudiments of Heyting valued set theory of Takeuti and Titani $[13,15]$ and intuitionistic linear algebra of Ruitenberg [11].

\subsection{Intuitionistic Set Theory}

By $Z_{F}$ we mean a first-order intuitionistic theory with a unary relation symbol $E$ and two binary relation symbols $\in$ and $=$ satisfying the following nonlogical axioms:

(A1) Equality axioms: $u=u$,

$$
u=v \rightarrow v=u,
$$




$$
u=v \wedge \varphi(u) \rightarrow \varphi(v) \text {, and }(\mathbb{E} u \vee \mathbb{E} v \rightarrow u=v) \rightarrow u=v .
$$

(A2) Extensionality: $\dot{\forall} z(z \in u \leftrightarrow z \in v) \wedge(E u \leftrightarrow E v) \rightarrow u=v$.

(A3) Pairing: $\dot{\exists} z \dot{\forall} x(x \in z \leftrightarrow x=u \vee x=v)$.

(A4) Union: $\dot{\exists} v \dot{\forall} x(x \in v \leftrightarrow \dot{\exists} y \in u(x \in y))$.

(A5) Power sets: $\dot{j} v \dot{\forall} x(x \in v \leftrightarrow \dot{\forall} y \in u(y \in x))$.

(A6) $\in$-induction: $\dot{\forall} x(\dot{\forall} y \in x \varphi(y) \rightarrow \varphi(x)) \rightarrow \dot{\forall} x \varphi(x)$.

(A7) Infinity: $\dot{\exists} v(\dot{\exists} x \in v \wedge \dot{\forall} x \in v \dot{\exists} y \in v(x \in y))$.

(A8) Separation: $\dot{\exists} v \dot{\forall} x(x \in v \leftrightarrow x \in u \wedge \varphi(x))$.

(A9) Collection: $\dot{\exists} v(\dot{\forall} x \in u \dot{\exists} y \varphi(x, y) \rightarrow \dot{\forall} x \in u \dot{\exists} y \in v \varphi(x, y))$.

In the above list $\dot{\forall} x \cdots$ and $\dot{\exists} x \cdots$ are abbreviations of $\forall x(E x \rightarrow \cdots)$ and $\exists x$ $(E x \wedge \cdots)$. Since $\forall x$ and $\exists x$ will usually appear in these forms, we will often write $\forall x$ and $\exists x$ simply for $\dot{\forall} x$ and $\dot{\exists} x$.

\subsection{Heyting Valued Mlodels}

Let $V$ be a standard universe of $\mathrm{ZFC}$ and let $\Omega$ be a complete Heyting algebra. For each ordinal $\alpha$ we define $V_{\alpha}^{(\Omega)}$ inductively to be the set of all ordered pairs $\langle u, E u\rangle$ such that:

(1) $E u \in \Omega$;

(2) $u$ is an $\Omega$-valued function defined on a subset $\mathscr{D}(u)$ of $V_{\beta}^{(\Omega)}$ for some ordinal $\beta<\alpha$;

(3) $\forall x \in \mathscr{D}(u)(u(x) \leqq E u \wedge E x)$.

Now $V^{(\Omega)}$ is defined to be the class $\cup_{\alpha \in O n} V_{a}^{(\Omega)}$, which is to be called an $(\Omega$ valued) sheaf model, can be considered to be a Heyting valued model of $\mathbb{Z} \mathbb{F}_{I}$ by defining $[E u \rrbracket$ with

(1) $\llbracket E u \rrbracket=E u$,

and by defining $\llbracket u \in v \rrbracket$ and $\llbracket u=v \rrbracket$ with the following simultaneous induction

(2) $[u \in \in v]]=\vee y \in \mathscr{D}(v)(v(y) \wedge \llbracket u=y \rrbracket)$,

(3) $\llbracket u=v \rrbracket=\wedge x \in \mathscr{D}(u)(u(x) \rightarrow \llbracket x \in v \rrbracket \wedge \wedge y \in \mathscr{D}(v)(v(y) \rightarrow \llbracket y \in u \rrbracket) \wedge(E u \leftrightarrow$ $E v)$,

and then by assigning a Heyting value $\llbracket \varphi \rrbracket$ to each nonatomic sentence $\varphi$ inductively as follows:

(4) $\llbracket \varphi_{1} \wedge \varphi_{2} \rrbracket=\llbracket \varphi_{1} \rrbracket \wedge \llbracket \varphi_{2} \rrbracket$,

(5) $\llbracket \varphi_{1} \vee \varphi_{2} \rrbracket=\llbracket \varphi_{1} \rrbracket \vee \llbracket \varphi_{2} \rrbracket$,

(6) $\llbracket \varphi_{1} \rightarrow \varphi_{2} \rrbracket=\llbracket \varphi_{1} \rrbracket \rightarrow \llbracket \varphi_{2} \rrbracket$, 
(7) $\llbracket \backslash \varphi \rrbracket=7 \llbracket \varphi \rrbracket$,

(8) $\llbracket \exists x \varphi(x) \rrbracket=\vee x \in V(\Omega) \llbracket \varphi(x) \rrbracket$,

(9) $\llbracket \forall x \varphi(x) \rrbracket=\vee x \in V^{(\Omega)} \llbracket \varphi(x) \rrbracket$.

Now we have

Theorem 2.2.1. $V^{(\Omega)}$ is a model of $\mathrm{ZF}_{\mathrm{I}}$.

The class $V$ can be embedded into $V^{(\Omega)}$ by transfinite induction as follows:

$$
\check{y}=\{\langle\grave{x}, 1\rangle \mid x \in y\} \text { and } E \grave{y}=1 \text { for } y \in V .
$$

For $u \in V^{(\Omega)}$ and $p \in \Omega$, we define $u\left\lceil p\right.$ to be the element of $V^{(\Omega)}$ such that $\mathscr{D}(u\lceil p)=\{x\lceil p \mid x \in \mathscr{D}(u)\}$,

$\left(u\lceil p)\left(x\lceil p)=\vee\left\{u(t) \wedge p \mid t \in \mathscr{D}(u), t\left\lceil p=x \Gamma^{-} p\right\} \quad\right.\right.\right.$ for $\quad x \in \mathscr{D}(u)$, and $E(u\lceil p)=E u \wedge p$.

In the sequel we implicitly identify $x, y \in V^{(\Omega)}$ time and again provided $\llbracket x=y \rrbracket=1$.

\subsection{Sheaves over Complete Heyting Algebras}

A presheaf over a complete Heyting algebra $\Omega$ is a triple $\langle S, E, \Gamma\rangle$ of a set $S$ and two functions $E: S \rightarrow \Omega$ and $\Gamma: S \times \Omega \rightarrow S$ with the following properties:

(1) $a\lceil 0=b\lceil 0$,

(2) $a \Gamma E a=a$,

(3) $E(a\lceil p)=E a \wedge p$,

(4) $(a\lceil p)\lceil q=a \Gamma(p \wedge q)$.

For convenience we often say simply that $S$ is a presheaf over $\Omega$ without mentioning $E$ and $\Gamma$ explicitly. Members $a, b$ of a presheaf $S$ over $\Omega$ are said to be compatible whenever $a\lceil E b=b\lceil E a$. A subset $F$ of $S$ whose members are pairwise compatible is called compatible. A presheaf $S$ over $\Omega$ is called a sheaf over $\Omega$ if for any compatible subset $F$ of $S$ there exists a unique $g \in S$ such that:

(1) $f \in F$ implies $g\lceil E f=f$,

(2) $E g=\vee\{E f \mid f \in F\}$.

The subset $\{a \in S \mid E a=p\}$ is denoted by $\Gamma(p, S)$.

Theorem 2.3.1. For any $\tilde{u} \in V^{(\Omega)}, u=\left\{x \in V^{(\Omega)} \mid \llbracket x \in u \rrbracket=E x\right\}$ is a sheaf 
to be called the sheaf represented by $u$. Conversely, for any sheaf $S$ over $\Omega$, there is an element $u \in V^{(\Omega)}$ such that the sheaf $\tilde{u}$ represented by $u$ is isomorphic to $S$.

Similarly we have

Theorem 2.3.2. Let $u_{1}, u_{2} \in V^{(\Omega)}$. Then any function $f: u_{1} \rightarrow u_{2}$ in $V^{(\Omega)}$ renders a unique function $\tilde{f}: \tilde{u}_{1} \rightarrow \tilde{u}_{2}$ such that for each $a \in \tilde{u}_{1}$,

$$
E_{\tilde{u}_{1}} a=E_{\tilde{u}_{2}} \tilde{f}(a)=\llbracket\langle a, \tilde{f}(a)\rangle \in f \rrbracket .
$$

This gives a bijective correspondence between functions $f: u_{1} \rightarrow u_{2}$ in $V^{(\Omega)}$ and functions $g: \tilde{u}_{1} \rightarrow \tilde{u}_{2}$ such that

$$
\begin{aligned}
& E_{\tilde{u}_{1}} a=E_{\tilde{u}_{2}} g(a) \text { and } g(a)\lceil p=g(a\lceil p) \\
& \text { for any } p \in \Omega \text { and } a \in \tilde{u}_{1} .
\end{aligned}
$$

\subsection{Intunationistic Limear Algebra}

Definition $\left(Z_{I}\right)$. A relation $\neq$ on a set $S$ is called apartness if for any $a, b, c \in S$, we have:

(1) $a \neq b \rightarrow b \neq a$;

(2) $a=b \leftrightarrow \neg(a \neq b)$;

(3) $a \neq b \rightarrow a \neq c \vee b \neq c$.

Defimition $\left(\mathbb{Z F}_{\mathrm{I}}\right)$. A ring $S$ with apartness $\neq$ is called an apartness ring if for $a, b, c \in S$, we have:

(1) $a \neq b \rightarrow a+c \neq b+c$;

(2) $a \neq b \wedge c \neq 0 \rightarrow a c \neq b c$.

Defimition $\left(\mathbb{Z F}_{\mathrm{I}}\right)$. An apartness ring $S$ with 1 is called an apartnes field if for any $a \in S$, we have:

(1) $1 \neq 0$;

(2) $a \neq 0 \rightarrow \exists a^{-1}\left(a^{-1} \neq 0 \wedge a a^{-3}=1\right)$.

Definition $\left(\mathrm{ZF}_{\mathrm{I}}\right)$. Let $F$ be an apartness field. Then an $F$-module $T$ with apartness $\neq$ is called an apartness vector space over $F$ provided for any $a, a^{\prime} \in F$ and any $u, u^{\prime}, v, v^{\prime} \in T$, we have:

(1) $a u \neq a^{\prime} u^{\prime} \rightarrow a \neq a^{\prime} \bigvee u \neq u^{\prime}$;

(2) $u+v \neq u^{\prime}+v^{\prime} \rightarrow u \neq u^{\prime} \vee v \neq v^{\prime}$.

Definition $\left(\mathrm{ZF}_{\mathrm{I}}\right)$. Let $T$ be an apartness vector space over an apartness 
field $F$. A finite set $\left\{e_{1}, \cdots, e_{n}\right\}$ of vectors is called an apartness basis if

(1) $\left\{e_{1}, \cdots, e_{n}\right\}$ is apartness linearly independent, i.e., $\forall\left\{a_{1}, \cdots, a_{n}\right\} \subset F\left(a_{i} \neq 0 \rightarrow a_{1} e_{1}+\cdots+a_{n} e_{n} \neq 0\right) ;$

(2) $T$ is generated by $\left\{e_{1}, \cdots, e_{n}\right\}$, i.e., $\forall u \in T \exists\left\{a_{1}, \cdots, a_{n}\right\} \subset F\left(u=a_{1} e_{1}+\cdots+a_{n} e_{n}\right)$.

Theorem 2.401 $\left(Z_{F_{I}}\right)$. Let $T$ be an apartness vector space over an apartness field $F$. If $\left\{e_{1}, \cdots, e_{m}\right\}$ and $\left\{e_{1}^{\prime}, \cdots, e_{n}^{\prime}\right\}$ are bases for $T$, then $m=n$.

Definition $\left(Z_{\mathrm{I}}\right)$. Let $T_{1}$ and $T_{2}$ be apartness vector spaces over an apartness field $F$. A mapping $\varphi: T_{1} \rightarrow T_{2}$ is called a homomorphism if

$$
\forall u, v \in T_{1} \forall a, b \in F(\varphi(a u+b v)=a \varphi(u)+b \varphi(v)) .
$$

A homomorphism $\varphi: T_{1} \rightarrow T_{2}$ is called an embedding if

$$
\forall u, v \in T_{1}(\varphi(u)=\varphi(v) \rightarrow u=v) .
$$

A homomorphism $\varphi: T_{1} \rightarrow T_{2}$ is called an apartness embedding if

$$
\forall u, v \in T_{1}(u \neq v \rightarrow \varphi(u) \neq \varphi(v)) .
$$

An (apartness) embedding $\varphi: T_{1} \rightarrow T_{2}$ is called an (apartness) isomorphism if

$$
\forall v \in T_{2} \exists u \in T_{1}(\varphi(u)=v) \text {. }
$$

\section{5. $\mathbb{R}^{(\Omega)}$ and $\mathbb{R}^{\circ 0}$}

Let $V$ be a standard universe of ZFC and let $\Omega$ be the topology of a smooth manifold $B$. Then the set $\mathbb{R}^{(\Omega)}$ of all real numbers in $V^{(\Omega)}$ is defined to be the set of all ordered pairs $\langle L, U\rangle$ of subsets of $\stackrel{Q}{Q}$ such that

(1) $\exists r, s \in \mathscr{Q}(s \in L \wedge r \in U)$,

(2) $\forall r \in \mathscr{Q} \neg(r \in U \wedge r \in L)$,

(3) $\forall r \in \check{Q}(r \in U \leftrightarrow \exists s \in \check{Q}(s<r \wedge s \in U))$,

(4) $\forall r \in \grave{Q}(r \in L \leftrightarrow \exists s \in \mathscr{Q}(r<s \wedge s \in L))$,

(5) $\forall r, s \in \mathscr{Q}(s<r \rightarrow s \in L \vee r \in U)$.

We denote by $\mathbb{R}^{\infty}$ the subfield of $\mathbb{R}^{(\Omega)}$ whose representing sheaf is the sheaf of all real-valued smooth functions on open subsets of $B$, while the representing sheaf of $\mathbb{R}^{(\Omega)}$ is the sheaf of all real-valued continuous functions on open subsets of $B$. In most of the paper $\mathbb{R}^{\infty}$ plays a role of $\mathbb{R}$ internally, but we should note that $\mathbb{R}^{\infty}$ fails to be a real-like field. Indeed, $\mathbb{R}^{\infty}$ is an apartness subfield of $\mathbb{R}^{(\Omega)}$ 


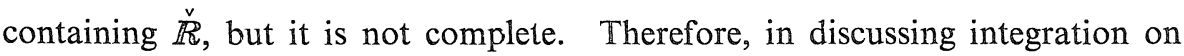
manifolds internally in Section 6, we have to make a detour through $\mathbb{R}^{(\Omega)}$.

\section{§3. The Imtermalization of Fibre Bundlles}

First of all, we recall some definitions in the arena of differentiable manifolds.

Definition $(\mathbb{Z F C})$. An n-dimensional topological manifold is a paracompact Hausdorff space $M$ whose every point has a neighborhood homeomorphic to an open subset of $\mathbb{R}^{n}$. In this case we write $n=\operatorname{dim} M$. A chart for the $n$ dimensional topological manifold $M$ is a triple $(U, u, V)$, where $U$ is an open subset of $M, V$ is an open subset of $\mathbb{R}^{n}$, and $u: U \rightarrow V$ is a homeomorphism. A chart $(U, u, V)$ is called cubic if $V$ is $C_{3}$, where $C_{\varepsilon}=\left\{\left(x^{1}, \cdots, x^{n}\right) \in \mathbb{R}^{n}|| x^{i} \mid<\varepsilon\right.$, $i=1, \cdots, n\}$ for each $\varepsilon>0$. A chart $(U, u, V)$ is often denoted simply by $(U, u)$, since $V$ is determined completely by the former data. An atlas on the $n$ dimensional topological manifold $M$ is a family of charts $\left\{\left(U_{\alpha}, u_{\infty}\right)\right\} \alpha \in \mathcal{A}$ with $M=\bigcup \alpha \in \mathcal{A} U_{\alpha}$.

Defimition (ZFC). Let $M$ be a topological manifold. Then an atlas

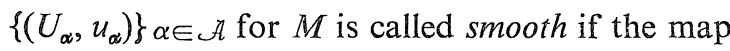

$$
u_{\alpha} \circ u_{\beta}^{-1}: u_{\beta}\left(U_{\infty} \cap U_{\beta}\right) \rightarrow u_{\alpha}\left(U_{\infty} \cap U_{\beta}\right)
$$

is smooth (i.e., infinitely differentiable) for any $\alpha, \beta \in \mathcal{A}$. Two smooth atlases are called equivalent if their union is again a smooth atlas. A smooth structure on $M$ is defined to be an equivalence class of smooth atlases on $M$. A topological manifold endowed with a smooth structure is called a smooth manifold. A chart of a smooth manifold will always mean a chart in a smooth atlas of the smooth structure.

Definaition $(Z F C)$. Let $M, N$ be smooth manifolds with their respective

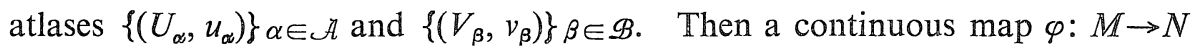
is called smooth if the map

$$
v_{\beta} \circ \varphi \circ u_{\alpha}^{-1}: u_{\alpha}\left(U_{\alpha} \cap \varphi^{-1}\left(V_{\beta}\right)\right) \rightarrow v_{\beta}\left(V_{\beta}\right)
$$

is smooth for any $\alpha \in \mathcal{A}, \beta \in \mathscr{B}$. A smooth map $\varphi: M \rightarrow N$ is called a diffeomorphism if it has a smooth inverse $\varphi^{-1}: N \rightarrow M$. Two smooth manifolds $M, N$ are called diffeomorphic if there exists a diffeomorphism $\varphi: M \rightarrow N$.

Definition (ZFC). Let $\pi: E \rightarrow B$ be a smooth map between smooth mani- 
folds. The map $\pi$ is said to have the local product property with respect to a smooth manifold $F$ if there is an open covering $\left\{U_{\alpha}\right\} \alpha \in \mathcal{A}$ of $B$ with a family $\left\{\varphi_{\alpha}\right\} \alpha \in \mathcal{A}$ of diffeomorphisms

$$
\varphi_{\alpha}: U_{o s} \times F \rightarrow \pi^{-1}\left(U_{\alpha}\right)
$$

such that

$$
\pi \varphi_{\alpha}(x, y)=x
$$

for any $x \in U_{\alpha}, y \in F$. The system $\left\{\left(U_{\alpha}, \varphi_{\alpha}\right)\right\}_{\alpha \in \mathcal{A}}$ is called a local decomposition of $\pi$. A smooth fibre bundle is a quadruple $(E, \pi, B, F)$, where $\pi: E \rightarrow B$ is a smooth map of the local product property with respect to a smooth manifold $F$. A local decomposition for $\pi$ is called a coordinate representation for the fibre bundle. In the fibre bundle $(E, \pi, B, F)$ the spaces $E, B$ and $F$ are called the total space, the base space and the typical fibre respectively. We will often say simply that $E$ is a smooth fibre bundle provided the other three components $\pi, B$ and $F$ are clear from the context. A cross-section of a fibre bundle $(E, \pi$, $B, F)$ on an open subset $U$ of $B$ is a smooth map $\sigma: U \rightarrow E$ such that $\pi \circ \sigma=\iota_{U}$. We denote by $\Gamma(U)$ the totality of cross-sections on $U$.

Now we recall some definitions and results of internal topologies.

Definition $\left(\mathbb{Z F}_{\mathrm{I}}\right)$. A set $\mathcal{O}(X)$ of subsets of $X$ is called a topology on $X$ if $X \in \mathcal{O}(X)$ and $\mathcal{O}(X)$ is a cHa with respect to binary intersection and arbitrary union. A special set $X$ endowed with topology $\mathcal{O}(X)$ is called a topological space. A topological space $X$ with apartness $\neq$ is called a separated space if

(1) $\forall x \in X(\{y \in X: y \neq x\} \in \mathcal{O}(X))$, and

(2) $\forall U \in \mathcal{O}(X) \forall x \in U \forall y \in X(y \in U \vee y \neq x)$.

A separated space $X$ is said to be Hausdorff if

$$
\forall x, y \in X(x \neq y \rightarrow \exists U, V \in \mathcal{O}(X)(x \in U \wedge y \in V \wedge(U \cap V=\phi))) .
$$

Definnition $(Z F C)$. Let $\Omega$ be a topology $O(B)$ and $X$ be a separated space in $V^{(\Omega)}$. We define a topological space $\bar{X}$ from $X$ as follows:

$$
\bar{X}=\{(t, x) \in B \times \bar{X} \mid t \in \llbracket x \in X \rrbracket\} / \sim,
$$

where $(t, x) \sim\left(t^{\prime}, x^{\prime}\right)$ iff $t=t^{\prime} \notin \llbracket x \neq x^{\prime} \rrbracket$. We denote the equivalence class containing $(t, x)$ by $\langle t, x\rangle$. For each $U \in \widetilde{\mathcal{O}(X)}$, the corresponding subset $\bar{U}$ of $\bar{X}$ is defined as follows: 


$$
\bar{U}=\{\langle t, x\rangle \in \bar{X} \mid t \in \llbracket x \in U \rrbracket\} .
$$

Set $\mathcal{O}(\bar{X})=\{\bar{U} \subset \bar{X} \mid U \in \widetilde{\mathcal{O}(X)}\}$.

Theorem 3.1 (ZFC) (Takeuti and Titani [15]). If $\Omega$ is a topology $\Theta(B)$ and $X$ is a separated space in $V^{(\Omega)}$, then $\bar{X}$ is a iopological space with topology $\mathcal{O}(\bar{x})$. In particular, $\overline{\mathbb{R}}^{\infty}=B \times \mathbb{R}$.

Theorem 3.2 (ZFC) (Takeuti and Titani [15]). Let st be a topology $\mathcal{O}(B)$ of a Hausdorff space $B$ and $X$ be a separated space in $V^{(\Omega)}$. Then $X$ is a Hausdorff space in $V^{(\Omega)}$ iff $\bar{X}$ is a Hausdorff space.

Defininition $\left(\mathbb{Z F}_{\mathrm{I}}\right)$. Let $X$ and $Y$ be topological spaces. Then a function $f: X \rightarrow Y$ is continuous if $\forall U \in \mathcal{O}(Y)\left(f^{-1}(U) \in \mathcal{O}(X)\right)$.

Theorem 3.3 (ZFC) (Takeuti and Titani [15]). Let \& be a topology $O(B)$. If $X, Y$ are separated spaces and $f: X \rightarrow Y$ is a continuous function in $V^{(\Omega)}$, then the function $\bar{f}: \bar{X} \rightarrow \bar{Y}$ defined by

$$
\bar{f}\langle t, x\rangle=\langle i, \tilde{f}(x)\rangle
$$

is continuous. Furthermore, if $f$ is an apartness homeomorphism in $\mathbb{V}^{(\Omega)}$ (i.e., $\llbracket \forall x, x^{\prime} \in \mathbb{X}\left(x \neq x^{\prime} \rightarrow f(x) \neq f\left(x^{\prime}\right)\right) \wedge \forall y \in Y \exists x \in X(f(x)=y) \wedge f^{-1}: Y \rightarrow X$ is continuous $\rrbracket=1$ ), then $\bar{f}: \bar{X} \rightarrow \bar{Y}$ is a homeomorphism.

Defimition $\left(\mathbb{Z} F_{I}\right)$. Let $X$ be a topological space. A subset $\left\{U_{i}\right\} i \in g$ of $\mathcal{O}(X)$ is called an open covering of $X$ if $U_{i \in g} U_{i}=X$. An open covering $\left\{U_{i}\right\}_{i \in g}$ is said to be locally finite if there exists an open covering $\left\{U_{j}\right\} j \in \mathcal{g}$ of $X$ such that the set $\left\{i \in \mathcal{g} \mid U_{i} \cap U_{j} \neq \phi\right\}$ is finite for each $j \in \mathcal{g}$. The space $X$ is called paracompact if for every open covering $\left\{U_{i}\right\} i \in g$ of $X$ there exist a locally finite open covering $\left\{U_{j}\right\} j \in \mathcal{g}$ of $X$ and a function $\varphi: g \rightarrow g$ such that $U_{j} \subset U_{\varphi(j)}$ for any $j \in g$.

Theorem 3.4 (ZFC) (Takeuti and Titani [15]). Let $B$ be a smooth manifold with topology $\Omega=\mathcal{O}(B)$. Then a separated space $X$ in $\mathbb{V}^{(\Omega)}$ is paracompact iff $\bar{X}$ is paracompact.

$\mathbb{D}$ efinaicion $(Z \mathrm{FC})$. Let $B$ be a smooth manifold with topology $\Omega=\mathcal{O}(B)$. Then the internal notion of a smooth manifold and the like within $V^{(\Omega)}$ are defined as in the previous definition merely with apparent modifications (i.e., $\mathbb{R}$ should be replaced by $\mathbb{R}^{\infty}$, due consideration must be paid to apartness, etc.). 
Now we are ready to internalize fibre bundles.

Theorem 3.5 (ZFC). Let $(E, \pi, B, F)$ be a smooth fibre bundle with $\&$ being the topology $\mathcal{O}(B)$ of the base space $B$. We define $E^{\infty}$ to be the set in $V^{(\Omega)}$ whose representing sheaf is

$$
U \in \mathcal{O}(B) \mapsto \Gamma(U)
$$

Then the set $E^{\infty}$ can naturally be made a smooth manifold in $V^{(\Omega)}$ and $\overline{\mathbb{E}}^{\infty}=E$. For each open subset $U$ of $E$ we write $U^{\infty}$ for the corresponding open subset of $E^{\infty}$ in $V^{(\Omega)}\left(\right.$ i.e., $\left.\bar{U}^{\infty}=U\right)$.

Proof. This is merely a special and rather easy case of Takeuti and Titani $[15 ; \S 3.5]$.

\section{§4. The Internalization of Vector Bundles over Fibre Bundlles}

First of all, we recall

Definition (ZFC). A vector bundle is a quadruple $\xi=(E, \pi, B, F)$, where

(1) $\xi$ is a smooth fibre bundle;

(2) the fibres $F_{x}=\pi^{-1}(x)$ for $x \in B$ are real linear spaces;

(3) $F$ is a real linear space of the form $\mathbb{R}^{n}$ ( $n$ is called the rank of $\xi$ );

(4) there is a coordinate representation $\left\{\left(U_{\alpha}, \varphi_{\alpha}\right)\right\} \alpha \in \mathcal{A}$ such that the maps $\varphi_{\alpha, x}: \mathbb{R}^{n} \rightarrow F_{x}$ defined by $\varphi_{\alpha}(x, y)$ for $\alpha \in U_{\alpha}$ and $y \in \mathbb{R}^{n}$ are linear isomorphisms.

A coordinate representation for $\xi$ which satisfies the above condition (4) is called a coordinate representation for the vector bundle $\xi$. We will often say that $E$ is a vector bundle (over $B$ ) provided the other three components are clear from the context.

Defimition (ZFC). Let $B$ be a smooth manifold with topology $\Omega=\mathcal{O}(B)$. Then the internal notion of a vector bundle in $V^{(\Omega)}$ can be defined as in the previous definition merely with obvious modifications.

The following construction of vector bundles is well-known and highly useful.

Theorem 4.1 (ZFC).

(1) Let $B$ be a smooth manifold with an open covering $\left\{U_{a}\right\} \alpha \in \mathcal{A}$. Let us suppose also that a family $\left\{\psi_{\alpha \beta}\right\}_{\alpha, \beta} \in \mathcal{A}$ of smooth functions $\psi_{\alpha \beta}: U_{\alpha} \cap U_{\beta} \rightarrow$ 
$\mathrm{GL}(n ; \mathbb{R})$ is given and satisfies the following condition (in this case the family $\left\{\psi_{\alpha \beta}\right\} \alpha, \beta \in \mathcal{A}$ is called a system of (n-dimensional) transition functions with respect to the open covering $\left.\left\{U_{\alpha}\right\} \alpha \in \mathcal{A}\right)$ :

(*)

$$
\psi_{\alpha \gamma}(x)=\psi_{\alpha \beta}(x) \psi_{\beta \gamma}(x) \text { for any } \alpha, \beta, \gamma \in \mathcal{A}, x \in U_{\alpha} \cap U_{\beta} \cap U_{\gamma} \text {. }
$$

Then the quotient set of the disjoint union $\cup \alpha \in \mathcal{A}\left(U_{\omega} \times \mathbb{R}^{n}\right)$ by the following equivalence relation $\sim$ can be made a vector bundle over $B$ in the natural way; for $(x, a) \in U_{a} \times \mathbb{R}^{n}$ and $(y, b) \in U_{\beta} \times \mathbb{R}^{n}$,

$$
(x, a) \sim(y, b) \text { iff } x=y \in U_{\alpha} \cap U_{\beta} \text { and } a=\psi_{\alpha \beta}(x) b .
$$

This vector bundle is called the vector bundle obiained from $\left\{\psi_{\alpha \beta \beta}\right\} \alpha, \beta \in \mathcal{A}$ by pasting.

(2) Conversely, let $\left(E, \pi, B, \mathbb{R}^{n}\right)$ be a vector bundle with a coordinate representation $\left\{\left(U_{\alpha}, \varphi_{\alpha}\right)\right\} \alpha \in \mathcal{A}$ for the vector bundle. Then the fanily $\left\{\varphi_{\alpha \beta}: U_{\alpha} \cap U_{\beta}\right.$ $\rightarrow \mathrm{GL}(n ; \mathbb{R})\}$ with $\varphi_{\alpha \beta}(x)=\varphi_{\alpha, x}^{-1} \circ \varphi_{\beta, x}$ for $x \in U_{\alpha} \cap U_{\beta}$ satisfies the above condition (*) and the original vecior bundle can be ic'entified with the vector bundle obtained from $\left\{\varphi_{\alpha \beta}\right\} \alpha, \beta \in \mathcal{A}$ by pasting.

Theorem $4.2(\mathbb{Z F C})$. Let $\mathbb{B}$ be a smooth manifold with topology $\Omega=O(B)$. Then Theorem 4.1 with obvious modifications still holds within $V^{(\Omega)}$.

Theorem $4.3(\mathbb{E F C})$. Let $(E, \pi, \mathbb{B}, F)$ be a smooth fibre bundle with $\Omega$ being the topology $O(B)$ of the base space $\mathbb{B}$. Then $\mathbb{E}^{\infty}$ is a smooth manifold in $V^{(\Omega)}$, as we have discussed in Seciion 3. Let us suppose further that a vector bundle $\left(Z, \rho, \mathbb{E}, \mathbb{R}^{n}\right)$ with $E$ as its base space is given. We define the set $Z^{\infty}$ in $V^{(\Omega)}$ whose representing sheaf is

$$
U \in \mathcal{O}(B) \mapsto \Gamma^{\prime}(U),
$$

where $\Gamma^{\prime}(U)=\left\{f: U \rightarrow Z \mid f\right.$ is smooih and $\left.\pi \circ \rho \circ f=\iota_{U}\right\}$. We also define a funcm tion $\rho^{\infty}: Z^{\infty} \rightarrow E^{\infty}$ in $V^{(\Omega)}$ such that

$$
\widetilde{\rho^{\infty}}(f)=\rho \circ f \text { for } f \in \Gamma^{\prime}(U) .
$$

Then they form a vector bundle $\left(Z^{\infty}, \rho^{\infty}, E^{\infty},\left(\mathbb{R}^{\infty}\right)^{n}\right)$ in $V^{(\Omega)}$.

Proof. Follows from the definitions.

Theorem $\mathbb{A}_{0} \Delta(\mathbb{Z F C})$. Let $(\mathbb{E}, \pi, B, F)$ be a smooih fibre bundle with $\Omega$ being the topology $\mathcal{O}(B)$ of the base space $B$. Let $\left\{U_{a}\right\} \alpha \in \mathcal{A}$ be an open covering of $B$, with respect to which a system of transition functions $\left\{\psi_{\alpha \beta}: U_{a} \cap U_{\beta} \rightarrow\right.$

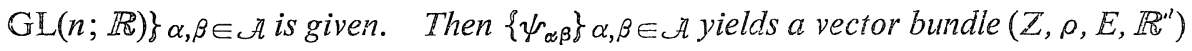


by pasting, as we have discussed in Theorem 4.1. This vector bundle then renders a vector bundle $\left(Z^{\infty}, \rho^{\infty}, E^{\infty},\left(\mathbb{R}^{\infty}\right)^{n}\right)$ in $V^{(\Omega)}$, as we have discussed in Theorem 4.3. By the way, the family $\left\{\psi_{\alpha \beta}\right\} \alpha, \beta \in \mathcal{A}$ yields a system $\left\{\psi_{\alpha \beta \beta}^{\infty}\right\} \alpha, \beta \in \mathcal{H}$ of transition functions with respect to the open covering $\left\{U_{\alpha}^{\infty}\right\} \alpha \in \mathcal{H}$ in $V^{(\Omega)}$, where $\widetilde{\psi}_{\alpha \beta}^{\infty}(f)=$ $\psi_{\alpha \beta} \circ f$ for any $f \in \widetilde{U_{\alpha}^{\infty}} \cap \widetilde{U_{\beta}^{\infty}}$. Then we conclude that the vector bundle obtained from $\left\{\psi_{\alpha_{\beta}}^{\infty}\right\} \alpha, \beta \in \mathcal{A}$ by pasting can be identified with the above vector bundle $\left(Z^{\infty}\right.$, $\left.\rho^{\infty}, E^{\infty},\left(\mathbb{R}^{\infty}\right)^{n}\right)$ in $V^{(\Omega)}$.

Proof. Follows from the definitions.

Now we would like to apply this general consideration to tangent bundles and orientation bundles.

Definition (ZFC). Let $M$ be an $n$-dimensional smooth manifold with a smooth atlas $\left\{\left(U_{\alpha}, u_{\alpha}\right)\right\}_{\alpha \in \mathcal{A}}$. Then it is easy to see that the family $\left\{\psi_{\alpha \beta}: U_{\alpha} \cap\right.$ $\left.U_{\beta} \rightarrow \mathrm{GL}(n ; \mathbb{R})\right\} \alpha, \beta \in \mathcal{H}$, where $\psi_{\alpha \beta}(x)=D\left(u_{\alpha} \circ u_{\beta}^{-1}\right)\left(u_{\beta}(x)\right)$ for $x \in U_{\alpha} \cap U_{\beta}$ and $D$ denotes the so-called Jacobian matrix, forms a system of transition functions with respect to the open covering $\left\{U_{\alpha}\right\} \alpha \in \mathcal{A}$ and, by pasting, gives rise to the tangent bundle $T(M)$ of $M$.

$\mathbb{D e f i n i t i o n}$ (ZFC). Let $B$ be a smooth manifold with topology $\Omega=\mathcal{O}(B)$. Then the internal notion of the tangent bundle of a smooth manifold in $V^{(\Omega)}$ can be defined as in the previous definition only with obvious modifications.

Defimition (ZFC). Let $(E, \pi, B, F)$ be a smooth fibre bundle with $m$-dim $B$ and $n=\operatorname{dim} F$. We can choose a smooth atlas $\left\{\left(U_{\alpha}, u_{\alpha}\right)\right\} \alpha \in \mathcal{H}$ for the base space $B$ and a coordinate representation $\left\{\left(U_{\alpha}, \varphi_{\alpha}\right)\right\}_{\alpha \in \mathcal{A}}$ for the fibre bundle with a common open covering $\left\{U_{\alpha}\right\} \alpha \in \mathcal{A}$. Let $\left\{\left(V_{i}, v_{i}\right)\right\} i \in g$ be a smooth atlas for the typical fibre $F$. We define a diffeomorphism

$$
f_{\alpha, i}: \varphi_{\alpha}\left(U_{\alpha} \times V_{i}\right) \rightarrow u_{\alpha}\left(U_{\alpha}\right) \times v_{i}\left(V_{i}\right)
$$

as $\left(u_{\alpha} \times v_{i}\right) \circ \varphi_{\alpha}^{-1}$ for $\alpha \in \mathcal{A}, i \in \mathcal{G}$. We then define a diffeomorphism

$$
f_{\alpha, i, \beta, j}: f_{\beta, j}\left(\varphi_{\alpha}\left(U_{\alpha} \times V_{i}\right) \cap \varphi_{\beta}\left(U_{\beta} \times V_{j}\right)\right) \rightarrow f_{\alpha, i}\left(\varphi_{\alpha}\left(U_{\alpha} \times V_{i}\right) \cap \varphi_{\beta}\left(U_{\beta} \times V_{j}\right)\right)
$$

as $f_{\alpha, i} \circ f_{\beta, j}^{-1}$ for $\alpha, \beta \in \mathcal{A}, i, j \in \mathcal{g}$. Since $D f_{\alpha, i, \beta, j}$ is of the form

$$
\left[\begin{array}{cc}
D_{11} f_{\alpha, i, \beta, j} & 0 \\
D_{21} f_{\alpha, i, \beta i j} & D_{22} f_{\alpha, i, \beta, j}
\end{array}\right]
$$

with 


$$
\begin{aligned}
& D_{11} f_{\alpha, i, \beta, j} \in \mathrm{GL}(m ; \mathbb{R}), \\
& D_{21} f_{\alpha, i, \beta, j} \in M(n, m ; \mathbb{R}), \text { and } \\
& D_{22} \int_{\alpha, i, \beta, j} \in \mathrm{GL}(n ; \mathbb{R}),
\end{aligned}
$$

and the Jacobian matrix of the composition of functions is the composition of the Jacobian matrices of respective functions, the family

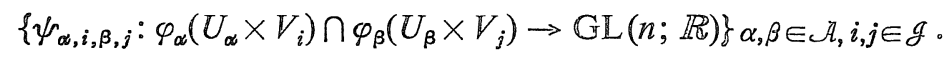

where $\psi_{\alpha, i, \beta, j}(x)=D_{22} f_{\alpha, i, \beta, j}\left(f_{\beta, j}(x)\right)$ for $x \in \varphi_{\alpha}\left(U_{\alpha} \times V_{i}\right) \cap \varphi_{\beta}\left(U_{\beta} \times V_{j}\right)$, forms a system of transition functions with respect to the open covering $\left\{\varphi_{\alpha}\left(U_{\alpha} \times\right.\right.$ $\left.\left.V_{i}\right)\right\} \alpha \in \mathcal{A}, i \in g$ of $E$ and gives rise to a vector bundle over $\mathbb{E}$, to be called the vertical tangent bundle of $E$ and to be denoted by $T^{V}(E)$.

The equivalence between the above definition of the vertical tangent bundle and another popular definition of the vertical subbundle of the tangent bundle $T(E)$ of $E$ as the kernel of $d \pi: T(E) \rightarrow T(B)$, which is easy and belongs completely to standard mathematics, is left to the reader.

Now we are ready to present one of the main results of this section.

Theorem 4.5 (ZFC). Let $(E, \pi, E, F)$ be a smooth fibre bundle with $\Omega$ being the topology $\mathcal{O}(B)$ of $B$. Then the tangent bundle of $E^{\infty}$ in $V^{(\Omega)}$ is externally the vertical tangent bundle of $E$.

Proof. Once we notice that the system of transition funcions in the definition of a vertical tangent bundle gives rise to a system of transition functions for the tangent bundle of $E^{\infty}$ in $V^{(\Omega)}$, the theorem follows directly from Theorem 4.4 .

Now we would like to deal with another important class of vector bundles, say, orientation bundles.

Defingition (ZFC). Let $M$ be a smooth manifold with a smooth atlas $\left\{\left(U_{\alpha}, u_{\alpha}\right)\right\}_{\alpha \in \mathcal{A}}$. Then it is easy to see that the family $\left\{\psi_{\alpha \beta \beta}: U_{a i} \cap U_{\beta} \rightarrow \mathbb{G L}\right.$

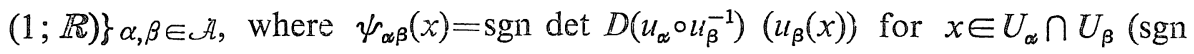
stands for sign and det stands for determinant), forms a system of transition functions with respect to the open covering $\left\{U_{\alpha}\right\} \alpha \in \mathcal{A}$ and, by pasting, gives rise to a vector bundle to be called the orientation bundle of $M$ and to be denoted by $\Theta(M)$.

Definition (ZFC). Let $B$ be a smooth manifold with topology $\Omega=\mathcal{O}(B)$. Then the notion of the orientation bundle of a smooth manifold in $V^{(\Omega)}$ can be 
defined as in the previous definition with obvious modifications.

Definnition ( $Z F C)$. Under the same assumptions and notations in the definition of vertical tangent bundle the family

$$
\left\{x_{\alpha, i, \beta, j}: \varphi_{\alpha}\left(U_{\alpha} \times V_{i}\right) \cap \varphi_{\beta}\left(U_{\beta} \times V_{j}\right) \rightarrow \mathbb{G L}(1 ; \mathbb{R})\right\} \alpha, \beta \in \mathcal{A}, i, j \in \mathcal{g}
$$

with $x_{\alpha, i, \beta, j}(x)=\operatorname{sgn} \operatorname{det} D_{22} f_{\alpha, i, \beta, j}\left(f_{\beta, j}(x)\right)$ for $x \in \varphi_{\alpha}\left(U_{\alpha} \times V_{i}\right) \cap \varphi_{\beta}\left(U_{\beta} \times V_{i}\right)$ forms a system of transition functions with respect to the open covering

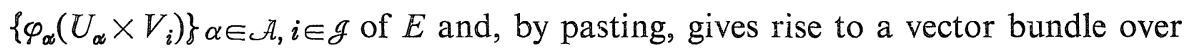
$E$ to be called the vertical orientation bundle of $E$ and to be denoted by $\Theta^{V}(E)$.

Theorem $A 6(\mathbb{Z} F C)$. Let $(E, \pi, B, F)$ be a smooth fibre bundle with $\Omega$ being the topology $\mathcal{O}(B)$ of the base space $B$. Then the orientation bundle of $E^{\infty}$ in $V^{(\Omega)}$ is externally the vertical orientation bundle of $E$.

Proof. Similar to Theorem 4.5.

Definition (ZFC). Let $M$ be a smooth manifold. Then a nowhere vanishing cross-section of $\Theta(M)$ on $M$ is called an orientation of $M$.

Definition (ZFC). Let $B$ be a smooth manifold with topology $\Omega=\mathcal{O}(B)$. Then the internal notion of an orientation of a smooth manifold in $V^{(\Omega)}$ can be defined as in the previous definition.

Defininition (ZFC). Let $(E, \pi, B, F)$ be a smooth fibre bundle. A nowhere vanishing cross-section of $\Theta^{V}(E)$ on $E$ is called an orientation of the smooth fibre bundle.

Theorem $4.7(Z \mathrm{ZFC})$. Let $(\mathbb{E}, \pi, B, F)$ be a smooth fibre bundle with $\&$ being the topology $\mathcal{O}(B)$ of $B$. Then every orientation of the smooth fibre bundle gives rise to an orientation $\mathrm{D}^{\infty}$ of $E^{\infty}$ in $V^{(\Omega)}$ such that

$$
\widetilde{\mathfrak{0}^{\infty}}(f)=\sigma \circ f \text { for any } f \in \widetilde{E^{\infty}} \text {. }
$$

Proof. Follows from the definitions.

Remark (ZFC). Let $M$ be a smooth manifold. Then two orientations $\mathfrak{D}, \mathfrak{D}^{\prime}$ are said to be equivalent if $\mathfrak{D}(x)=\lambda(x) \mathfrak{D}^{\prime}(x)$ with $\lambda(x)>0$ for any $x \in M$. Equivalent orientations are considered to determine the same orientation. A chart $(U, u)$ of an oriented smooth manifold $M$ is called oriented if the standard orientation of $u(U)$ is equivalent to the orientation induced from that of $M$. These notions apply also internally. 


\section{§5. Differential Forms}

Definition (ZFC). Let $I V$ be a smooth manifold. The fibre $\mathbb{T}_{x}(M)$ of $T(M)$ at $x \in M$ is called the tangent space of $M$ at $x$. For each natural number $p$, the $p$ th exterior powers $\wedge^{p} \mathbb{T}_{x}^{*}(M)$ of the dual space $\mathbb{T}_{x}^{*}(M)$ of the tangent space $T_{x}(M)$ of $M$ at $x$ for all $x \in M$ together form a vector bundle $\wedge^{p} T^{*}(M)$ over $M$ in the well-known way. We denote by $A^{p}(M)$ the toiality of crosssections of $\wedge^{p} T^{*}(M)$ on $M$, whose elements are called differential forms of degree $p$ on $M$ (or simply $p$-forms on $M$ ). The value $\omega_{x}$ of a $p$-form $\omega \in A^{p}(M)$ at $x \in M$ can be considered an alternating $p$-linear form on $T_{x}(M)$. We define $A(M)$ to be the direct sum of $A^{p}(M)$ 's for all $p \geq 0$.

Let $E$ be a finite-dimensional real vector space. Then $\left(\wedge^{p} T_{x}^{*}(M)\right) \otimes E^{\prime}$ s for all $x \in M$ together form a vector bundle $\left(\wedge^{p} T^{*}(M)\right) \otimes E$ over $M$, whose cross-sections on $M$ are called E-valued differential forms of degree $p$ on $M$ (or simply $E$-valued $p$-forms on $M$ ). The totality of $E$-valued $p$-forms on $M$ is denoted by $A^{p}(M ; E)$. The value $\omega_{x}$ of $\omega \in A^{p}(M ; E)$ at $x \in M$ can be considered an alternating $p$-linear function with values in $\mathbb{E}$. We define $A(M ; E)$ to be the direct sum of $A^{p}(M ; E)$ 's for all $p \geq 0$.

Definition $(Z F C)$. Let $B$ be a smooth manifold with topology $\Omega=O(B)$. Then the internal notion of a differential form on a smooth manifold in $V^{(\Omega)}$ and the like can be given as in the previous definition with obvious modifications.

Let $E$ be a finite-dimensional apartness vector space in $V^{(\Omega)}$. Then the internal notion of an $\mathbb{E}$-valued differential form on a smooth manifold in $V^{(\Omega)}$ and the like can be given as in the previous definition.

Theorem 5.1 (ZFC). Lei $\xi=(E, \pi, E, F)$ be a smooth fibre bundle, $\zeta=$ $\left(Z, \rho, B, \mathbb{R}^{m}\right)$ be a vector bundle with the same base $B$, and $T^{V}(E)$ be the vertical tangent bundle of $\xi$ with projection $\sigma: \mathbb{T}^{V}(E) \rightarrow E$. Lei $\Phi: \wedge^{n} \mathbb{T}^{V}(E) \rightarrow \zeta$ be a bundle map (i.e., smooth, fibre-preserving and linear on each fibre), inducing $\pi: E \rightarrow B$ in the base manifolds. Let $\Omega$ be the topology $\mathcal{O}(B)$ of $\mathbb{B}$. Then bundle map $\Phi$ naturally gives rise to an $n$-form $\omega$ on $\mathbb{E}^{\infty}$ in $V^{(\Omega)}$ such that

$$
\widetilde{\omega}_{\mu}\left(\eta^{1}, \cdots, \eta^{n}\right)=\mathscr{\Phi} \circ\left(\eta^{1} \wedge \cdots \wedge \eta^{n}\right)
$$

for any $\mu \in \widetilde{E^{\infty}}, \eta^{i} \in \widetilde{T^{V}(E)^{\infty}}(1 \leq i \leq n)$ satisfying $\sigma \circ \eta^{i}=\mu(1 \leq i \leq n)$. This differential form $\omega$ is denoted by $\Phi^{\infty}$.

Proof. Follows from the definitions.

Theorem 5.2 (ZFC). Let $B$ be a smooth manifold with topology $\Omega=\mathcal{O}(B)$. 
Then there exists a smooth function f on $C_{3}$ in $V^{(\Omega)}$ such that:

(1) $0 \leq \mathrm{f} \leq 1$;

(2) $f(x)=1$ for any $x \in C_{1}$;

(3) $f(x)=0$ for any $1\left(x \in C_{2}\right)$.

Proof. The existence of such a function $f$ in the external world is wellknown. The desired $f$ can be obtained as $f^{\infty}: C_{3} \rightarrow \mathbb{R}^{\infty}$ in $V^{(\Omega)}$ such that

$$
\tilde{f}^{\infty}(g)=f \circ g \text { for } g \in \tilde{C}_{3} .
$$

Now we would like to demonstrate the existence and uniqueness of exterior differentiation internally.

Theorem 5.3 (ZFC). Let $B$ be a smooth manifold with topology $\Omega=\mathcal{O}(B)$. Let $M$ be a smooth manifold in $V^{(\Omega)}$. Then there exists a unique $R^{\infty}$-linear map $d_{M}: A(M) \rightarrow A(M)$ such that:

(1) if $f \in A^{0}(M)=C^{\circ}(M)$ (the totality of smooth functions on $M$ ), then $d_{M} f=$ df (the differential of $f$ whose definition can be given as usual);

(2) if $\varphi \in A^{r}(M)$ and $\psi \in A^{s}(M)$, then

$$
d_{M}(\varphi \wedge \psi)=d_{M} \varphi \wedge \psi+(-1)^{r} \varphi \wedge d_{M} \psi
$$

(3) $d_{M}^{2}=0$.

Proof. Now that Theorem 5.2 is available, the proof is essentially the same as the standard one (see, e.g., Boothby [2; Chap. V, Theorem (8.1)].

We will often abbreviate $d_{M}$ to $d$. Since $A(M ; E)=A(M) \otimes E$ with $E$ a finite-dimensional apartness vector space, exterior differentiation on $A(M ; E)$ can naturally be defined as $d_{M} \otimes \iota_{E}$, which we also denote by $d_{M}$ or $d$.

Remark (ZFC). In the rest of this paper we will freely use some standard notations on differential forms and the like both externally and internally. For example, let $\varphi: M \rightarrow N$ be a smooth map between smooth manifolds and $f$ be a smooth function on $N$. Then the pull-back of $f$ by $\varphi$ is defined as $f \circ \varphi$ and denoted by $\varphi^{*}(f)$. Similarly, the pull-back $\varphi^{*}(\omega)$ of a differential form $\omega$ on $N$ by $\varphi$ can be defined both externally and internally.

Remark (ZFC). Let $M$ and $N$ be smooth manifolds. Then we have the decomposition $\mathbb{T}_{(x, y)}(M \times N)=\mathbb{T}_{x}(M) \times \mathbb{T}_{y}(N)$ for any $(x, y) \in M \times N$, which yields the derived decomposition $A(M \times N)=\sum_{p, q} A^{p, q}(M \times N)$. For example, if $\varphi \in A^{p}(M)$ and $\psi \in A^{q}(N)$, then $\pi_{M}^{*} \varphi \wedge \pi_{N}^{*} \psi \in A^{p, q}(M \times N)$, where $\pi_{M}: M \times N \rightarrow$ 
$M$ and $\pi_{N}: M \times N \rightarrow N$ are the canonical projections. For these matters, the reader is referred to Greub et al. [5; pp. 121-122].

Remark (ZFC). Let $M$ and $N$ be smooth manifolds again. The exterior differentiation $d_{M \times N}: A(M \times N) \rightarrow A(M \times N)$ can be decomposed into the partial exterior differentiations $d_{M}$ and $d_{N}$ with respect to $M$ and $N$ respectively, i.e., $d_{M \times N}=d_{M}+d_{N}$, for which the reader is referred to Greub et al. [5; pp. 148-149].

\section{§6. Imtegration on $\mathbb{N}$ I2mi̊lolds}

$\mathbb{D e n i m i t i o n ~}\left(\mathbb{Z F}_{\mathrm{I}}\right)$. A separated space $X$ is called compact if every open covering has a finite subcovering.

Theorem 6.1 (ZFC). Let $B$ be a locally compact Hausdorff space and $\Omega$ be its topology $\mathcal{O}(B)$. Then a separated space $X$ in $V^{(\Omega)}$ is compact iff $\bar{X}_{C}$ is compact for every compact subset $\mathbb{C}$ of $B$, where we write $\bar{X}_{D}=\{\langle t, x\rangle \mid t \in D$, $x \in \tilde{X}\}$ for $D \subset B$.

Proof. Suppose that $X$ is compact in $V^{(\Omega)}$ and that $\bar{X}_{C}$ is covered by open subsets $\left\{\bar{U}_{i}\right\} i \in \mathcal{g}$ of $\bar{X}$ for a compact subset $C$ of $B$. Then there exists an open covering $\left\{P_{\alpha}\right\} \alpha \in \mathcal{A}$ of $C$ such that $\bar{X}_{C \cap P_{\alpha}}$ is covered by a finite subcovering $\left\{\bar{U}_{i}\right\} i \in g_{\alpha}$ for each $\alpha \in \mathcal{A}$. Since $C$ is compact, we can assume without loss of generality that $\mathcal{A}$ is a finite set. Then the family $\left\{\bar{U}_{i} \mid i \in \mathcal{G}_{\alpha}, \alpha \in \mathcal{A}\right\}$ is a desired finite covering of $\bar{X}_{C}$. Conversely, suppose that $\bar{X}_{C}$ is compact for every compact subset $C$ of $B$ and that $P=\llbracket\left\{U_{i}\right\} i \in g$ is a covering of $X \rrbracket$. Then $\left\{\bar{U}_{i}\right\}_{i \in \tilde{g}}$ is a covering of $\bar{X}_{P}$. Since $B$ is locally compact Hausdorff, every point $x \in P$ has an open subset $P^{\prime}$ and a compact subset $C$ such that $x \in P^{\prime} \subset$ $C \subset P$. Since $\bar{X}_{C}$ is compact by assumption, we have $\mathbb{P}^{\prime} \leq \mathbb{L}\left\{U_{i}\right\} i \in g$ has a finite subcovering of $X \rrbracket$. Thus the proof is complete.

Theorem $\sigma_{0} 2(\mathrm{ZFC})$. Let $(E, \pi, B, F)$ be a smooth manifold with $\Omega$ being the topology $\mathcal{O}(B)$ of $B$. Then, by assigning to each continuous function $f: E^{\infty} \rightarrow$ $\mathbb{R}^{\infty}$ the composition of $\bar{f}: E=\overline{E^{\infty}} \rightarrow \overline{\mathbb{R}^{\infty}}=B \times \mathbb{R}$ and the projection of $B \times \mathbb{R}$ to the second argument (the resulting composition is denoted by $\hat{f}$ ), we obtain a bijective correspondence between continuous functions from $E^{\infty}$ io $\mathbb{R}^{\infty}$ in $\mathbb{V}^{(\Omega)}$ and continuous functions from $\mathbb{E}$ to $\mathbb{R}$.

Proof. Use a similar discussion to Takeuti [12; Chap. 3, §3] or Rousseau $[10 ; 2.2]$.

Defimition $(Z F C)$. Let $B$ be a smooth manifold with topology $\Omega=\mathcal{O}(B)$. 
Let $M$ be a smooth manifold in $V^{(\Omega)}$. Then a continuous function $f: M \rightarrow \mathbb{R}^{\infty}$ in $V^{(\Omega)}$ is said to be of compact support if there exists a finite family $\left\{\left(U_{i}, u_{i}\right)\right\} i \in g$ of cubic charts such that

$$
\forall x \in M\left(f(x) \neq 0 \rightarrow \exists i \in \mathcal{g}\left(x \in u_{i}^{-1}\left(C_{1}\right)\right)\right) .
$$

We recall

Defimition $(Z F C)$. Let $(E, \pi, B, F)$ be a smooth fibre bundle and $f: E \rightarrow \mathbb{R}$ be a continuous function. Then $f$ is said to be of fibre-compact support if for every compact subset $C \subset B$, the intersection $\pi^{-1}(C) \cap$ supp $f$ is compact, where $\operatorname{supp} f$ denotes the closure of $\{x \in E \mid f(x) \neq 0\}$.

The preceding two definitions can be applied to differential forms and the like. These two definitions, one of which is internal and the other of which is external, are related in the following theorem.

Theorem $6.3(Z F C)$. Let $(E, \pi, B, F)$ be a smooth fibre bundle with $\Omega$ being the topology $\mathcal{O}(B)$ of $B$. Then, under the bijective correspondence of Theorem 6.2 , a continuous function $f: E^{\infty} \rightarrow \mathbb{R}^{\infty}$ in $V^{(\Omega)}$ is of compact support iff $\hat{f}$ is of fibre-compact support.

Proof. Follows from the definitions and Theorem 6.1.

To define integration on smooth manifolds in $V^{(\Omega)}$, we begin with integration on $\left(\mathbb{R}^{\infty}\right)^{n}$.

Theorem $6.4(Z F C)$. Let $B$ be a smooth manifold with topology $\Omega=\mathcal{O}(B)$. Then a continuous function $f:\left(\mathbb{R}^{\infty}\right)^{n} \rightarrow \mathbb{R}^{\infty}$ in $V^{(\Omega)}$ is uniformly continuous on any $C_{\varepsilon}(\varepsilon>0)$.

Proof. This follows from Theorem 6.2 and the well-known theorem of standard mathematics that any real-valued continuous function on a compact metric space is uniformly continuous.

Defimition (ZFC). Let $B$ be a smooth manifold with topology $\Omega=\mathcal{O}(B)$. Let $f:\left(\mathbb{R}^{\infty}\right)^{n} \rightarrow \mathbb{R}^{\infty}$ be a continuous function in $V^{(\Omega)}$ with the property that there exists $C_{\varepsilon}$ such that $f(x)=0$ for any $x \notin C_{\varepsilon}$. Then, due to Theorem 6.4 , the usual definition of Riemann integral works well with respect to the real-like field $\mathbb{R}^{(\Omega)}$ and the value $\int f(x) d x^{1} \ldots d x^{n}$ is defined as an element of $\mathbb{R}^{(\Omega)}$, just as Takeuti [12; Chap. 3, §1] did for $n=1$.

Fortunately we have 
Theorem $6.5(Z F C)$. Under the same assumptions and notations of the above definition, we have

$$
\int f(x) d x^{1} \cdots d x^{n} \in \mathbb{R}^{\infty} .
$$

Proof. Follows from the well-known Leibniz's rule on differentiation of integrals with parameters (see, e.g., Dieudonné [16; (8.11.2)]).

Definition $(Z \mathbb{Z F C})$. Let $B$ be smooth manifold with topology $\Omega=\mathcal{O}(B)$. Let $M$ be an oriented $n$-dimensional smooth manifold in $V^{(\Omega)}$. Let $\omega$ be an $n$ form with compact support on $M$ in $V^{(\Omega)}$. I.e., there exists a finite family $\left\{\left(U_{i}, u_{i}\right)\right\} i \in \mathcal{I}$ of oriented cubic charts in $V^{(\Omega)}$ such that

$$
\forall x \in M\left(\omega_{x} \neq 0 \rightarrow \exists i \in \mathcal{g}\left(x \in u_{i}^{-1}\left(C_{1}\right)\right)\right) .
$$

Let $\uparrow$ be the function in Theorem 5.2. For each $i \in g$, let $g_{i}=u_{i}^{*}(f)=\left\{\circ u_{i}\right.$, which can be regarded as a smooth function on $N I$ in the natural way (extension by zero). The $n$-form $\left(\mathfrak{g}_{i} / \sum_{i \in g} \mathfrak{g}_{i}\right) \omega$ can be deiined safely and denoted by $\omega_{i}$. Let $\varphi_{i}$ be the $n$-form $\left(u_{i}^{-1}\right)^{*} \omega_{i}$ on $\mathbb{C}_{3}$, which is of the form $h_{i} d x^{1} \wedge \cdots \wedge d x^{n}$. The function $h_{i}$ can be considered to be defined on $\left(\mathbb{R}^{\infty}\right)^{n}$ in the natural way (extension by zero). Then we define $\int_{M} \omega$ to be

$$
\sum_{i \in \mathcal{g}} \int h_{i}(x) d x^{1} \ldots d x^{n} .
$$

Of course we need to verify

Theorem $6.6(Z F C)$. The above definition of $\int_{M} \omega$ is independent of a particular choice of oriented cubic charts.

Proof. Let $\left\{\left(V_{j}, v_{j}\right)\right\}_{j \in \mathcal{g}}$ be another such finite family of oriented cubic charts. Let $\mathfrak{g}_{j}=v_{j}^{*}(f)$ for each $j \in \mathcal{g}$. Let $\omega_{j}$ be $\left(\mathfrak{g}_{j} / \Sigma_{j \in g} \mathfrak{g}_{j}\right) \omega$ and $\varphi_{j}$ be $\left(v_{j}^{-1}\right)^{*} \omega_{j}$ for each $j \in \mathcal{G}$. The $n$-form $\varphi_{j}$ on $C_{3}$ is of the form $h_{j} d x^{1} \wedge \cdots \wedge d x^{n}$ for each $j \in \mathcal{g}$. Let $\omega_{i j}$ be $\left(g_{i} / \sum_{j \in g} g_{j}\right)\left(g_{j} / \sum_{j \in \mathcal{g}} g_{j}\right) \omega$ for each $i \in \mathcal{g}, j \in \mathcal{g}$. Let $\varphi_{i j}$ be the $n$-form $\left(u_{i}^{-1}\right)^{*} \omega_{i j}$, which is of the form $h_{i j} d x^{1} \wedge \cdots \wedge d x^{n}$ for each $i \in \mathcal{g}, j \in \mathcal{g}$. Let $\varphi_{j i}$ be the $n$-form $\left(v_{j}^{-1}\right)^{*} \omega_{i j}$, which is of the form $h_{j i} d x^{1} \wedge \cdots$ $\wedge d x^{n}$. By the way, the standard theorem on change of variables in Riemann integrals can be generalized easily to the case with parameters, which then renders an internalized version of the theorem on change of variables. I.e., we have

$$
\int h_{i j}(x) d x^{1} \circ d x^{n}=\int h_{j i}(x) d x^{1} \circ d x^{n} \text { 。 }
$$

Since $\quad \int h_{i}(x) d x^{1} \cdots d x^{n}=\sum_{j \in g} \int h_{i j}(x) d x^{1} \cdots d x^{n} \quad$ and $\quad \int h_{j}(x) d x^{1} \cdots d x^{n}=$ 
$\sum_{i \in \mathcal{g}} \int h_{j i}(x) d x^{1} \circ \cdot d x^{n}$, we have

$$
\begin{aligned}
& \sum_{i \in \mathcal{G}} \int h_{i}(x) d x^{1} \cdots d x^{n} \\
= & \sum_{\substack{i \in \mathcal{G} \\
j \in \mathcal{G}}} \int h_{i j}(x) d x^{1} \cdots d x^{n} \\
= & \sum_{\substack{i \in \mathcal{G} \\
j \in \mathcal{G}}} \int h_{j i}(x) d x^{1} \cdots d x^{n} \\
= & \sum_{j \in \mathcal{G}} \int h_{j}(x) d x^{1} \cdots d x^{n} .
\end{aligned}
$$

This completes the proof.

Definition $(Z F C)$. Let $\xi=(E, \pi, B, F)$ be an oriented smooth fibre bundle with $\operatorname{dim} F=n$ and $\zeta=\left(Z, \rho, B, \mathbb{R}^{m}\right)$ be a vector bundle with the same base space. Let us suppose further that a bundle map $\Phi: \wedge^{n} T^{V}(E) \rightarrow \zeta$ with fibre-compact support is given, inducing $\pi: E \rightarrow B$ in the base manifolds. For each $x \in B, \Phi$ determines a $\rho^{-1}(x)$-valued $n$-form $\Phi_{x}$ on $F_{x}=\pi^{-1}(x)$, say,

$$
\left(\Phi_{x}\right)_{y}\left(u_{1}, \cdots, u_{n}\right)=\Phi\left(u_{1} \wedge \cdots \wedge u_{n}\right)
$$

for $y \in \pi^{-1}(x), u_{i} \in T_{y}\left(F_{x}\right)=T_{y}^{V}(E)$. Since each $\Phi_{x}$ has compact support (this follows from the assumption that $\Phi$ has fibre-compact support) and the orientation of $\xi$ induces an orientation on each fibre $F_{x}$, we can define a map $\sigma$ : $B \rightarrow Z$ by

$$
\sigma(x)=\int_{F_{x}} \Phi_{x}
$$

for $x \in B$. Then it is well-known that $\sigma$ is a cross-section in $\zeta$, to be called the integral of $\Phi$ over the fibre.

Theorem $6.7(\mathbb{Z F C})$. Under the same assumptions and notations of the above definition, the vector $\int_{E^{\infty}} \Phi^{\infty} \in Z^{\infty}$ in $V^{(\Omega)}$ is externally the integral of $\Phi$ over the fibre, where $\Omega$ is the topology $O(B)$.

Proof. Follows from the definitions.

The following will be used in the succeeding section.

Definition $(Z F C)$. Let $(E, \pi, B, F)$ be an oriented smooth fibre bundle with $\operatorname{dim} F=n$. Let $\omega \in A_{F}^{n+p}(E)$ (=the totality of $(n+p)$-forms with fibrecompact support). Then $\omega$ determines naturally a bundle map $\Phi_{\omega}: \wedge^{n} T^{V}(E) \rightarrow$ $\wedge^{p} T^{*}(B)$, since for any $x \in E, u_{i}, v_{j}, w_{j} \in T_{x}^{V}(E)(1 \leq i \leq n, 1 \leq j \leq p)$, we have

$$
\omega_{x}\left(v_{1}, \cdots, v_{p}, u_{1}, \cdots, u_{n}\right)=\omega_{x}\left(w_{1}, \cdots, w_{p}, u_{1}, \cdots, u_{n}\right),
$$

provided $d \pi v_{j}=d \pi w_{j}(1 \leq j \leq p)$. We define 


$$
f_{F} \omega
$$

to be the integral of $\mathscr{D}_{\omega}$ over the fibre. Then the operator $f_{F}$ induces a linear map from $A_{F}^{n+p}(E)$ to $A^{p}(\mathbb{B})$. By decreeing that $f_{F} \omega=0$ for $\omega \in A_{F}^{q}(E)(\forall q<n)$, $f_{F}$ becomes a linear map from $A_{F}(E)$ (=the totality of differential forms on $E$ with fibre-compact support) to $A(B)$.

\section{§7. Stokes ${ }^{2}$ Theorem}

The first main result of this section is

Theorem 7.1 (ZFC). Let $B$ be a smooth manifold with topology $\Omega=\mathcal{O}(B)$. Let $M$ be an oriented n-dimensional smooth manifold in $V^{(\Omega)}$, on which a differential form $\omega$ of degree $n-1$ with compact suppori is given. Then we have

$$
f_{M} d \omega=0 \text {. }
$$

Proof. Since $\omega$ is of compact support by assumption, there exists a finite family of oriented cubic smooth charts $\left\{\left(U_{i}, u_{i}\right)\right\} i \in g$ such that $\forall x \in \mathbb{M}\left(\omega_{x} \neq 0\right.$ $\rightarrow \exists i \in \mathcal{g}\left(x \in u_{i}^{-1}\left(C_{1}\right)\right)$ ). Then the standard proof of Stokes' theorem (see, e.g., Boothby [2; pp. 257-258]) works well in $V^{(\Omega)}$, since the so-called fundamental theorem of calculus still holds internally (see, e.g., Takeuti [12; Chap. 3, Theorem 5]) and the iterated integration theorem can easily be internalized.

The above theorem can be generalized directly to vector-valued differential forms (i.e., differential forms with values in finite-dimensional apartness vector spaces).

Now we are ready to demonstrate that the following well-known theorem is the external form of an internalized version of Stokes' theorem in disguise.

Theorem $7.2(Z F C)$. Let $(E, \pi, B, F)$ be an oriented smooth fibre bundie. Then exierior differentiation and integration over the fibre commute, i.e.,

$$
d \circ f_{F}=f_{F} \circ d, \text { or exactly, } d_{B} \circ f_{F}=f_{F} \circ d_{M} \text {. }
$$

Proof. We should show that $d_{B}\left(f_{F} \omega\right)=f_{F}\left(d_{M} \omega\right)$ for any $\omega \in A_{F}(M)$. Since the statement is local with respect to $B$ and $\pi$ is of local product property, we can assume without loss of generality that $E=B \times F$. Since $A_{r}(B \times F)=$ $\sum_{p, q} A_{F}^{p, q}(B \times F)$ (sce, e.g., Greub el al. [5; pp. 12l-122]), we can also assume without loss of generality that $\omega \in A_{F}^{p, q}(B \times F)$. Since $d_{M}=d_{B}+d_{F}$ (see, e.g., Greub et al. [5; pp. 148-149]) and obviously

$$
d_{B}\left(f_{F} \omega\right)=f_{F}\left(d_{B} \omega\right),
$$


it suffices to show that

$$
f_{F}\left(d_{F} \omega\right)=0,
$$

which is nontrivial only in case of $\operatorname{dim} F=q+1$, when the equation follows directly from Theorem 7.1 as an external restatement.

Just as in standard mathematics, the internal notion of a smooth manifold and its related concepts can be generalized to that of a smooth manifold with boundary and its related correspondents. Then, as might be expected, we have

Theorem 7.3 (ZFC). Let $B$ be a smooth manifold with topology $\Omega=\mathcal{O}(B)$. Let $M$ be an oriented n-dimensional smooth manifold with boundary in $V^{(\Omega)}$, on which a differential form $\omega$ of degree $n-1$ with compact support is given. Then we have

$$
\int_{M} d \omega=(-1)^{n} \int_{\partial M} i^{*} \omega,
$$

where $\partial M$ is the boundary of $M$ with the induced orientation and $i: \partial M \rightarrow M$ is the inclusion mapping.

Proof. Essentially the same as the standard one (see, e.g., Boothby [2; pp. 257-258]). The major difference from that of Theorem 7.1 is only that we have to consider, besides cubic charts, the intersections of cubic charts with the upper half plane $H^{n}=\left\{x=\left(x^{1}, \cdots, x^{n}\right) \in\left(\mathbb{R}^{\infty}\right)^{n} \mid x^{n} \geq 0\right\}$.

The following well-known theorem of standard mathematics stands in the same relation to Theorem 7.3 as Theorem 7.2 does to Theorem 7.1.

Theorem 7.4 (ZFC). Let $\delta=(\mathbb{E}, \pi, B, F)$ be an oriented smooth fibre bundle with boundary. We denote the boundary of $F$ by $\partial F$, the boundary of $E$ by $\partial E$, and the ordinary smooth fibre bundle $\left(\partial E,\left.\pi\right|_{\partial E}, B, \partial F\right)$ by $\partial \delta$. Then we have

$$
f_{F} d \omega-d f_{F} \omega=(-1)^{k+n+1} f_{\partial F} i^{*} \omega
$$

for any $\omega \in A_{F}^{k}(E)$ (=the totality of differential forms on $E$ of degree $k$ with fibre-compact support), where $n=\operatorname{dim} F$ and $i: \partial E \rightarrow E$ is the inclusion mapping.

Proof. Proceed as in Theorem 7.2.

\section{§8. Open Problem (De Rham Cohomology)*}

Let $\xi=(E, \pi, B, F)$ be a smooth fibre bundle. We denote by $\Delta_{n}^{V}(E)$ the

* In this section we do not adhere to apartness. 
totality of bundle maps from $\wedge^{n} T^{V}(E)$ to the product bundle $B \times \mathbb{R}$ inducing $\pi: E \rightarrow B$ in the base manifolds. Then the exterior differentiation $d: \Delta_{n}^{V}(E) \rightarrow$ $\Delta_{n+1}^{V}(E)$ is defined, so that we can consider vertical cohomolgy groups $H_{n}^{V}(E)$. See, e.g., Greub et al. [5; p. 313].

By the way, as we have seen in Theorem 5.1, each $\mathscr{D} \in \mathcal{A}_{n}^{V}(\mathbb{E})$ gives rise to a differential form $\Phi^{\infty}$ on $E^{\infty}$ in $V^{(\Omega)}$, where $\Omega$ is the topology $\mathcal{O}(B)$ of $B$. Now we conjecture the following.

Compectrere $(\mathbb{Z F C})$. Let $\xi=(E, \pi, \mathbb{B} . F)$ be a smooth fibre bundle with $\Omega$ being the topology of $B$. Then the internal notion of the $n$-th de $\mathbb{R}$ ham cohomology group $H_{n}\left(E^{\infty}\right)$ in $V^{(\Omega)}$ is externally the sheaf determined by the presheaf

$$
U \in \Omega \mapsto H_{n}^{V}\left(\pi^{-1}(U)\right),
$$

where $H_{n}^{V}\left(\pi^{-1}(U)\right)$ is the vertical cohomology group for the smooth fibre bundle $\left.\xi\right|_{U}=\left(\pi^{-1}(U), \pi, U, F\right)$.

\section{References}

[1] Bishop, E. and Bridges, D., Consiructive analysis, Springer, Berlin, 1985.

[2] Boothby, W.M., An introduction to differenitable manifolds and Riemannian geometry, Academic Press, New York, 1975.

[3] Grayson, R.J., Concepts of general topology in constructive mathematics and in sheaves, Annals of Mathematical Logic, 201 (1981), 1-41.

[4] — Concepts of general topology in constructive mathematics and in sheaves, II, Annals of Mathernatical Logic, 23 (1982), 55-98.

[5] Greub, W., Halperin, S., and Vansione, R., Connections, curvature, and cohomology, I, Academic Press, New York, 1972.

[6] —- Connections, curvature, and cohomology, II, Academic Press, New York, 1973.

[7] Hattori, A., Differentiable manifolds (in Japanese), Iwanami, Tokyo, 1976.

[8] Matsushima, Y., Differeníiable manifolds, Marcel Dekker, New York, 1972.

[9] Nishimura, H., Heyting valued set theory and Sato hyperfunctions, Publ. RIMS, 22 (1986), 801-811.

[10] Rousseau, C., Topos theory and complex analysis, Lecture Notes in Mathematics, 753 (1977), Springer, Berlin, 623-659.

[11] Ruitenberg, W.B.G., Intuitionistic algebra, theory and sheaf models, dissertation, 1982.

[12] Takeuti, G., Intuitionistic set theory (in Japanese), Kinokuniya, Tokyo, 1980.

[13] Takeuti, G. and Titani, S., Heyting valued universes of intuitionistic set theory, Lecture Notes in Mathematics, 8DI (1980), Springer, Berlin, 189-306.

[14] — Globalization of intuitionistic set theory, preprini.

[15] —, Global intuitionistic analysis, preprini.

[16] Dieudonné, J., Foundations of modern analysis, Academic Press, New York, 1960.

[17] Shiga, K., Theory of differeniiable manifolds (in Japanese), 3 vols, Iwanami, Tokyo, 1976. 
